\title{
Stability of an SVI Epidemic Model
}

\author{
Md. Saiful Islam ${ }^{1}$, Dr. Md. Asaduzzaman ${ }^{2}$, Dr. Md. Nazrul Islam Mondal ${ }^{3}$ \\ ${ }^{1}$ Department of Computer Science and Engineering, Jatiya Kabi Kazi Nazrul Islam University, Trishal, \\ Mymensingh-2220, Bangladesh. \\ ${ }^{2}$ Department of Mathematics, University of Rajshahi, Rajshahi-6205, Bangladesh. \\ ${ }^{3}$ Department of Population Science and Human Resource Development, University of Rajshahi, Rajshahi-6205, \\ Bangladesh.
}

\begin{abstract}
The spread of communicable diseases is often described mathematically by compartmental models. A vaccine is a biological preparation that improves immunity to a particular disease. In this paper a nonlinear mathematical deterministic compartmental model for the dynamics of an infectious disease including the role of a preventive vaccine, natural birth rate and natural death rate is proposed and analyzed. The model has various kinds of parameter. We try to present a model for the transmission dynamics of an infectious disease and mathematically analyzed the stability of daisies free equilibrium and endemic equilibrium. Also we have given some strategy to control the epidemic by controlling the parameters.
\end{abstract}

Keywords: Stability analysis, Basic reproduction number, Diseases free equilibrium, Endemic equilibrium.

\section{Introduction}

Various kinds of deterministic models for the spread of infectious disease have been analyzed mathematically and applied to control the epidemic. Kermack and McKendrick proposed, as a particular case of a more general model presented in their seminal work [1]. Many epidemiological models have a disease free equilibrium (DFE) at which the population remains in the absence of disease [2]. The classical SIR models are very important as conceptual models (similar to predator-prey and competing species models in ecology). The SIR epidemic modeling yields the useful concept of the threshold quantity which determines when an epidemic occurs, and formulas for the peak infective fraction and the final susceptible fraction [3]. There are two major types of control strategies available to curtail the spread of infectious diseases: pharmaceutical interventions (drugs, vaccines etc) and non-pharmaceutical interventions (social distancing, quarantine). Vaccination is important for the elimination of infectious disease. Usually, the vaccination process are different schedules for different disease and vaccines. For some disease, such as hepatitis B virus infection, doses should be taken by vaccines several times and there must be some fixed time intervals between two doses. Vaccination, when it is available, is an effective preventive strategy. Arino et al introduced vaccination of susceptible individuals into an SIRS model and also considered vaccinating a fraction of newborns [4]. Buonomo et al studied the traditional SIR model with $100 \%$ efficacious vaccine [5]. Effective vaccines have been used successfully to control smallpox, polio and measles.

In this paper we consider an SI type model when a vaccination program is in effect and there is a constant flow of incoming immigrants or newborns. This paper basically the extension of "Stability analysis at DFE of an epidemic model in the presence of a preventive vaccine" [6]. Let $S(t)$ be the number of population who are susceptible to an infection at time $t, I(t)$ be the number of members who are infective at time $t$, and $V(t)$ be the number of members who are vaccinated at time $t$. The total population size at time $t$ is denoted by $N(t)$, with $N(t)=S(t)+V(t)+I(t)$. Assume that each infective makes $\alpha N$ contacts sufficient to transmit infection in unit time, where $\alpha$ is a constant. When an infective makes contact, the probability of producing a new infection is $S / N$, since the new infection can be made only when a contact is made with a susceptible individuals. Thus, the rate of producing new infections is $\alpha N \cdot \frac{S}{N} \cdot I=\alpha S I$. The susceptible population is vaccinated at a constant rate $\phi$. We assume that there is no disease related death but natural death, that is, 
unrelated to the disease is present. The population is replenished in two ways; birth and immigration. We assume that all newborns and immigrants enter the susceptible class at a constant rate $\Lambda$. In summary, the assumptions we have in this model is as follows:

$S(t), I(t), V(t)$ and $N(t)$ are the numbers of susceptible, infective, vaccinated, and total population at time $t$, respectively.

- $\quad$ There is a constant flow $\Lambda$ of new members into the susceptible population per unit time.

- The vaccine has effect of reducing infection by a factor of $\sigma$, so that $\sigma=0$ means that the vaccine is completely effective in preventing infection, while $\sigma=1$ means that the vaccine is utterly ineffective.

- The rate at which the susceptible population is vaccinated is $\phi$.

- $\quad$ There is a constant per capita natural death rate $\mu$ in each class.

- $\alpha N$ is the infectious contact rate per person in unit time.

The following table shows the summary of notation.

\begin{tabular}{|c|l|}
\hline Notation & Explanation \\
\hline$S(t)$ & Number of susceptible at time t \\
\hline$V(t)$ & Number of vaccinated individuals at time t \\
\hline$I(t)$ & Number of infective at time t \\
\hline$N(t)$ & Total number of population at time t \\
\hline$\Lambda$ & Birth rate \\
\hline$\alpha$ & Contact rate \\
\hline$\phi$ & Vaccination rate \\
\hline$\sigma$ & Factor by which the vaccine reduces infection \\
\hline$\mu$ & Natural death rate unrelated to the disease \\
\hline
\end{tabular}

Table -1: Summary of notation

\section{Model Formulation}

In our model, we have divided the population into three compartments (susceptible, vaccinated susceptible and infectious) depending on the epidemiological status of individuals. We denote the population of those who are susceptible as $S$, who are vaccinated susceptible as $V$ and those who subsequently infected as $I$. The model transfer diagram indicating the possible transitions between these compartments is shown in Figure 1.

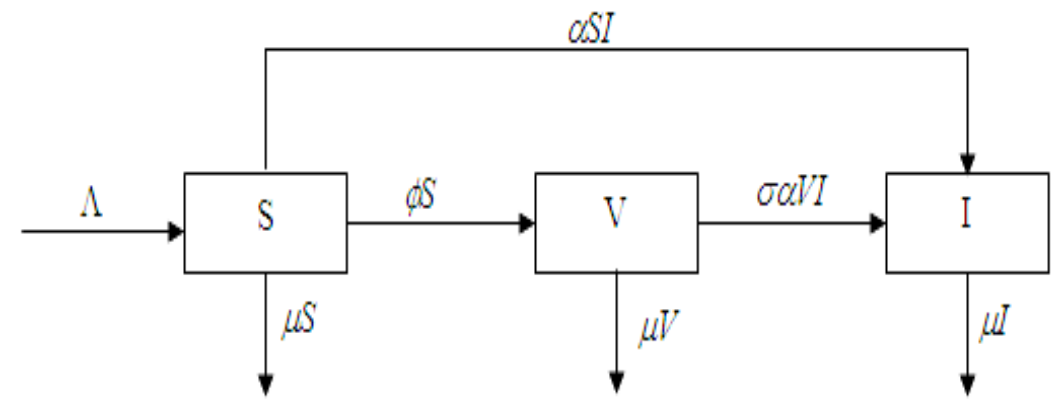

Figure-1: Diagram for SVI model

Populations enter the susceptible class at constant rate $\Lambda$. Natural death rate are assumed to be $\mu$. The population is assumed to undergo homogeneous mixing. We assume that each infective individual contacts an average number $\alpha$ with other individuals per unit time. Hence, the total number of contact by infective per unit time is $\alpha I$. Susceptible individuals are vaccinated at the rate $\phi$. Since the vaccine only provides partial protection to the infection, vaccinated individuals may still become infected but at the lower infection rate $\sigma \alpha$ 
than fully susceptible individuals. Here $1-\sigma \in[0,1]$ describes vaccine efficacy. When $\sigma=0$, the vaccine is perfectly effective and when $\sigma=1$, the vaccine has no effect at all on the immunity of vaccinated individuals. The differential equations of the model are given by:

$$
\left.\begin{array}{l}
\frac{d S}{d t}=\Lambda-\alpha S I-\phi S-\mu S \\
\frac{d V}{d t}=\phi S-\mu V-\sigma \alpha V I \\
\frac{d I}{d t}=\sigma \alpha V I+\alpha S I-\mu I
\end{array}\right\}
$$

\section{Equilibrium Conditions}

We can write the equilibrium conditions by letting the right hand side of equations of (1) to be zero. Thus the equilibrium conditions are

$$
\begin{gathered}
\Lambda-\alpha S I-\phi S-\mu S=0 \\
\phi S-\mu V-\sigma \alpha V I=0 \\
\sigma \alpha V I+\alpha S I-\mu I=0
\end{gathered}
$$

From (2) we get

$$
S=\frac{\Lambda}{\alpha I+\mu+\phi}
$$

Again from (3) we get

$$
V=\frac{\phi S}{\sigma \alpha I+\mu}
$$

$$
\Rightarrow \quad V=\frac{\phi \Lambda}{(\sigma \alpha I+\mu)(\alpha I+\mu+\phi)} \quad\left[\because S=\frac{\Lambda}{\alpha I+\mu+\phi}\right]
$$

Now from (4) factoring out the disease free equilibrium (DFE), we get

$$
I=0
$$

Then from (5) and (6), we get

$$
\begin{gathered}
S=\frac{\Lambda}{\mu+\phi} \\
V=\frac{\phi \Lambda}{\mu(+\mu+\phi)}
\end{gathered}
$$

and

Therefore the disease free equilibrium is

$$
P_{0}=\left(\frac{\Lambda}{\mu+\phi}, \frac{\phi \Lambda}{\mu(+\mu+\phi)}, 0\right)
$$

In order to study the stability of steady states we linearize (2), obtaining the Jacobean matrix.

$$
\begin{array}{r}
J=\left[\begin{array}{ccc}
\frac{\partial}{\partial S}\left(\frac{d S}{d t}\right) & \frac{\partial}{\partial V}\left(\frac{d S}{d t}\right) & \frac{\partial}{\partial I}\left(\frac{d S}{d t}\right) \\
\frac{\partial}{\partial S}\left(\frac{d V}{d t}\right) & \frac{\partial}{\partial V}\left(\frac{d V}{d t}\right) & \frac{\partial}{\partial I}\left(\frac{d V}{d t}\right) \\
\frac{\partial}{\partial S}\left(\frac{d I}{d t}\right) & \frac{\partial}{\partial V}\left(\frac{d I}{d t}\right) & \frac{\partial}{\partial I}\left(\frac{d I}{d t}\right)
\end{array}\right] \\
\Rightarrow \quad J=\left[\begin{array}{ccc}
-\alpha I-\phi-\mu & 0 & -\alpha S \\
\phi & -\mu-\alpha \sigma I & -\sigma \alpha V \\
\alpha I & \sigma \alpha I & \sigma \alpha V+\alpha S-\mu
\end{array}\right]
\end{array}
$$




\section{Stability of DFE.}

The Jacobean matrix at the DFE $P_{0}=\left(\frac{\Lambda}{\mu+\phi}, \frac{\phi \Lambda}{\mu(+\mu+\phi)}, 0\right)$ is

$$
J_{0}=\left[\begin{array}{ccc}
-\phi-\mu & 0 & -\frac{\alpha \Lambda}{\mu+\phi} \\
\phi & -\mu & -\frac{\sigma \alpha \phi \Lambda}{\mu(\mu+\phi)} \\
0 & 0 & \frac{\alpha \Lambda}{\mu+\phi}+\frac{\sigma \alpha \phi \Lambda}{\mu(\mu+\phi)}-\mu
\end{array}\right]
$$

$J_{0}$ has three real eigenvalues as follows

and

$$
\begin{gathered}
\lambda_{1}=-(\phi+\mu) \\
\lambda_{2}=-\mu \\
\lambda_{3}=\frac{\alpha \Lambda}{\mu+\phi}+\frac{\sigma \alpha \phi \Lambda}{\mu(\mu+\phi)}-\mu
\end{gathered}
$$

Since all parameters are positive then clearly $\lambda_{1}<0$ and $\lambda_{2}<0$, So the DFE is locally stable if and only if $\lambda_{3}<0$

Definition (Basic reproductive number): The basic reproductive number, $R_{0}$, is the expected number of secondary infections arising from a single individual during his or her entire infectious period, in the population of susceptible [7].

Lemma: The disease free equilibrium $p_{0}$ is locally stable if and only if $R_{0}<1$ where $R_{0}$ is the basic reproductive number.

Since the above linear system (1) is locally stable if and only if $\lambda_{3}<0$, i.e., $\frac{\alpha \Lambda}{\mu+\phi}+\frac{\sigma \alpha \phi \Lambda}{\mu(\mu+\phi)}<\mu$, i.e., $\frac{\alpha \Lambda}{\mu(\mu+\phi)}+\frac{\sigma \alpha \phi \Lambda}{\mu^{2}(\mu+\phi)}<1$. So by the above lemma the basic reproduction number $R_{0}=\frac{\alpha \Lambda}{\mu(\mu+\phi)}+\frac{\sigma \alpha \phi \Lambda}{\mu^{2}(\mu+\phi)}$

\section{Controlling The Epidemic}

Since for $R_{0}=\frac{\alpha \Lambda}{\mu(\mu+\phi)}+\frac{\sigma \alpha \phi \Lambda}{\mu^{2}(\mu+\phi)}<1$, there is no epidemic; we can take various steps to control the epidemic. If we consider $R_{0}$ as a function of $\sigma$. i.e, $R_{0}=R_{0}(\sigma)=\frac{\alpha \Lambda}{\mu(\mu+\phi)}+\frac{\sigma \alpha \phi \Lambda}{\mu^{2}(\mu+\phi)}=M_{1} \sigma+D_{1}$ where $M_{1}=\frac{\alpha \phi \Lambda}{\mu^{2}(\mu+\phi)}>0$ and $D_{1}=\frac{\alpha \Lambda}{\mu(\mu+\phi)}$. Therefore $R_{0}$ is a linear function of $\sigma$. Since $M_{1}>0$ (for positive parameters), So there exists a bifurcation value $\sigma_{0}=\frac{\mu^{3}+\mu^{2} \phi-\alpha \mu \Lambda}{\phi \alpha A}$ of $\sigma$ such that if $\sigma<\sigma_{0}$, then $R_{0}<1$ and if $\sigma>\sigma_{0}$, then $R_{0}>1$, i.e., if $\sigma<\sigma_{0}$, then the DFE $P_{0}$ is locally stable otherwise unstable (provided $\left.D_{1}<1\right)$. So if all parameters except $\sigma$ are constant, then we can control the epidemic by decreasing the value of $\sigma$ (increasing the vaccine efficiency) so that $\sigma<\sigma_{0}$. Again suppose $R_{0}$ is a function of $\alpha$. i.e, $R_{0}=R_{0}(\alpha)=\frac{\alpha \Lambda}{\mu(\mu+\phi)}+\frac{\sigma \alpha \phi \Lambda}{\mu^{2}(\mu+\phi)}=M_{2} \alpha$, where $M_{2}=\frac{\Lambda}{\mu(\mu+\phi)}+\frac{\sigma \phi \Lambda}{\mu^{2}(\mu+\phi)}>0$. Therefore $R_{0}$ is a linear function of $\alpha$. Since $M_{2}>0$ (for positive parameters), So there exists a bifurcation value $\alpha_{0}=\frac{\mu^{2}(\mu+\phi)}{\mu \Lambda+\sigma \phi \Lambda}$ of $\alpha$ such that if $\alpha<\alpha_{0}$, then $R_{0}<1$ and if $\alpha>\alpha_{0}$, then $R_{0}>1$, i.e., if $\alpha<\alpha_{0}$, then the DFE $P_{0}$ is locally 
stable otherwise unstable. Therefore if all parameters except $\alpha$ are constant, then we can control the epidemic by decreasing the value of $\alpha$ (decreasing the contact rate with infected individual) so that $\alpha<\alpha_{0}$. Similarly we can reduce the value of $R_{0}$ by decreasing the value of $\Lambda$ and we get a bifurcation value $\Lambda_{0}=\frac{\mu^{2}(\mu+\phi)}{\alpha \mu+\alpha \phi \sigma}$ of $\Lambda$ such that if $\Lambda<\Lambda_{0}$, then $R_{0}<1$ and if $\Lambda>\Lambda_{0}$, then $R_{0}>1$, i.e., if $\Lambda<\Lambda_{0}$, then the DFE $P_{0}$ is locally stable otherwise unstable. Therefore if all parameters except $\Lambda$ are constant, then we can control the epidemic by decreasing the value of $\Lambda$ so that $\Lambda<\Lambda_{0}$. Finally suppose $R_{0}$ is a function of $\phi$. i.e., $R_{0}=R_{0}(\phi)=\frac{\alpha \Lambda}{\mu(\mu+\phi)}+\frac{\phi \alpha \sigma \Lambda}{\mu^{2}(\mu+\phi)}$. So for positive parameters $\frac{d R_{0}}{d \phi}=\frac{\alpha(\sigma-1) \Lambda}{\mu^{3}+2 \phi \mu^{2}+\phi^{2} \mu}<0$. ( since $\left.\sigma<1\right)$ i.e., $R_{0}(\phi)$ is decreasing. We can reduce the value of $R_{0}$ by increasing the value of $\phi$. Therefore $\phi_{0}=\frac{\mu^{3}-\alpha \mu \Lambda}{\alpha \sigma \Lambda-\mu^{2}}$ is a bifurcation value of $\phi$ such that if $\phi>\phi_{0}$, then $R_{0}<1$ and if $\phi<\phi_{0}$, then $R_{0}>1$, i.e., if $\phi>\phi_{0}$, then the DFE $P_{0}$ is locally stable otherwise unstable. Therefore we can control the epidemic by increasing the value of $\phi$ so that $\phi>\phi_{0}$.

Discussion: In the above simulations we consider the initial value of infected individual is 1 , i.e., $I_{0}=1$. We see that if all parameters except $\sigma$ are fixed, there exist a bifurcation value $\sigma_{0}$. If $\sigma<\sigma_{0}$, then the number of infected individuals is decreasing as $t \rightarrow \infty$. On the other hand if $\sigma>\sigma_{0}$, then the number of infected individuals is increasing as $t \rightarrow \infty$. Similarly we see that if all parameters except $\alpha$ are fixed, there exist a bifurcation value $\alpha_{0}$. If $\alpha<\alpha_{0}$, then the number of infected individuals is decreasing as $t \rightarrow \infty$. On the other hand if $\alpha>\alpha_{0}$, then the number of infected individuals is increasing as $t \rightarrow \infty$. Again there exist a bifurcation value $\Lambda_{0}$. If $\Lambda<\Lambda_{0}$, then the number of infected individuals is decreasing as $t \rightarrow \infty$. On the other hand if $\Lambda>\Lambda_{0}$, then the number of infected individuals is increasing as $t \rightarrow \infty$. Finally there exist a bifurcation value $\phi_{0}$. If $\phi<\phi_{0}$, then the number of infected individuals is increasing as $t \rightarrow \infty$. On the other hand if $\phi>\phi_{0}$, then the number of infected individuals is decreasing as $t \rightarrow \infty$.

\section{Stability of Endemic Equilibrium}

Usually the stability analysis at endemic equilibrium (here $I>0$ ) is very difficult. Now solving (2), (3) and (4) we get

$$
\begin{gathered}
S=\frac{\mu(\sigma \alpha+\mu)}{\alpha(\sigma \phi+\sigma \alpha+\mu)} \\
V=\frac{\mu \phi}{\alpha(\sigma \phi+\sigma \alpha+\mu)} \\
I=\frac{\alpha(\sigma \phi+\sigma \alpha+\mu) \Lambda-\left(\sigma \alpha \mu+\sigma \alpha \phi+\mu^{2}+\mu \phi\right) \mu}{\alpha \mu(\sigma \alpha+\mu)}
\end{gathered}
$$

i.e., The endemic equilibrium is

provided

$$
P^{*}=\left(\frac{\mu(\sigma \alpha+\mu)}{\alpha(\sigma \phi+\sigma \alpha+\mu)}, \frac{\mu \phi}{\alpha(\sigma \phi+\sigma \alpha+\mu)}, \frac{\alpha(\sigma \phi+\sigma \alpha+\mu) \Lambda-\left(\sigma \alpha \mu+\sigma \alpha \phi+\mu^{2}+\mu \phi\right) \mu}{\alpha \mu(\sigma \alpha+\mu)}\right)
$$

$$
I=\frac{\alpha(\sigma \phi+\sigma \alpha+\mu) \Lambda-\left(\sigma \alpha \mu+\sigma \alpha \phi+\mu^{2}+\mu \phi\right) \mu}{\alpha \mu(\sigma \alpha+\mu)}>0
$$

Theorem (Routh-Hurwitz stability criterion [8]) : Given the characteristics polynomial

$$
P(\lambda)=\lambda^{n}+a_{1} \lambda^{n-1}+a_{2} \lambda^{n-2}+a_{3} \lambda^{n-3}+\ldots .+a_{n}
$$

where the coefficients $a_{i}$ are real constant for $i=1,2,3, \ldots . . n$, define the Hurwitz matrices using the coefficients 
$a_{i}$ of the characteristics polynomial as follows

$$
\begin{gathered}
H_{1}=\left[a_{1}\right] \\
H_{2}=\left[\begin{array}{ll}
a_{1} & 1 \\
a_{3} & a_{2}
\end{array}\right] \\
H_{3}=\left[\begin{array}{ccc}
a_{1} & 1 & 0 \\
a_{3} & a_{2} & a_{1} \\
a_{5} & a_{4} & a_{3}
\end{array}\right] \\
H_{n}=\left[\begin{array}{cccccc}
a_{1} & 1 & 0 & 0 & \cdots & 0 \\
a_{3} & a_{2} & a_{1} & 1 & \cdots & 0 \\
a_{5} & a_{4} & a_{3} & a_{2} & \cdots & 0 \\
\vdots & \vdots & \vdots & \vdots & \cdots & \vdots \\
0 & 0 & 0 & 0 & \cdots & a_{n}
\end{array}\right]
\end{gathered}
$$

where $a_{j}=0$ if $j>n$. All of the roots of the polynomial equation $P(\lambda)=0$ are negative or have negative real part iff the determinants of all Hurwitz matrices are positive.

i.e., $\operatorname{det}\left(H_{j}\right)>0$, for $j=1,2,3, \ldots \ldots . n$.

When $n=2$, the Routh-Hurwitz stability criterion simplify to

and

$$
\begin{gathered}
\operatorname{det}\left(H_{1}\right)=a_{1}>0 \\
H_{2}=\left[\begin{array}{cc}
a_{1} & 1 \\
0 & a_{2}
\end{array}\right]=a_{1} a_{2}>0
\end{gathered}
$$

or, $a_{1}>0$ and $a_{2}>0$. For polynomial of degree $n=2,3$ and 4 , the Routh-Hurwitz stability criterion is summarized as follows:

$$
\begin{aligned}
& n=2: a_{1}>0 \text { and } a_{2}>0 . \\
& n=3: a_{1}>0, a_{3}>0 \text { and } a_{1} a_{2}>a_{3} . \\
& n=4: a_{1}>0, a_{3}>0, a_{4}>0 \text { and } a_{1} a_{2} a_{3}>a_{3}^{2}+a_{1}^{2} a_{4} .
\end{aligned}
$$

In order to study the stability of steady states we consider the equilibrium conditions (2), (3), (4) and the Jacobean matrix

$$
J=\left[\begin{array}{ccc}
-\alpha I-\phi-\mu & 0 & -\alpha S \\
\phi & -\mu-\alpha \sigma I & -\sigma \alpha V \\
\alpha I & \sigma \alpha I & \sigma \alpha V+\alpha S-\mu
\end{array}\right]
$$

Using the condition (4), i.e., $\sigma \alpha V I+\alpha S I-\mu I=0$, i.e., $\sigma \alpha V+\alpha S-\mu=0$, we get

$$
J=\left[\begin{array}{ccc}
-\alpha I-\phi-\mu & 0 & -\alpha S \\
\phi & -\mu-\alpha \sigma I & -\sigma \alpha V \\
\alpha I & \sigma \alpha I & 0
\end{array}\right]
$$

Again from the equilibrium condition (2), we get

$$
\begin{gathered}
\Lambda-(\alpha I+\phi+\mu) S=0 \\
\Rightarrow \quad(\alpha I+\phi+\mu)=\frac{\Lambda}{S}
\end{gathered}
$$

Use the above value, we get 


$$
J=\left[\begin{array}{ccc}
-\frac{\Lambda}{S} & 0 & -\alpha S \\
\phi & -\mu-\alpha \sigma I & -\sigma \alpha V \\
\alpha I & \sigma \alpha I & 0
\end{array}\right]
$$

Again from the equilibrium condition (3), we get

$$
\Rightarrow \quad \begin{gathered}
\phi S-\mu V-\sigma \alpha V I=0 \\
-\mu-\sigma \alpha I=-\frac{\phi S}{V}
\end{gathered}
$$

Use the above value, we get

$$
J=\left[\begin{array}{ccc}
-\frac{\Lambda}{S} & 0 & -\alpha S \\
\phi & -\frac{\phi S}{V} & -\sigma \alpha V \\
\alpha I & \sigma \alpha I & 0
\end{array}\right]
$$

After calculating by wxMaxima we get the characteristic equation of the above matrix as

$$
\begin{gathered}
\lambda^{3}+\left(\frac{\Lambda}{S}+\frac{\phi S}{V}\right) \lambda^{2}+\left(\alpha^{2} \sigma^{2} V I+\frac{\phi \Lambda}{V}+\alpha^{2} S I\right) \lambda+\frac{\alpha^{2} \sigma^{2} \Lambda V I}{S}+\frac{\phi \alpha^{2} S^{2} I}{V}+\phi \alpha^{2} \sigma S I=0 \\
\Rightarrow \quad \lambda^{3}+a_{1} \lambda^{2}+a_{2} \lambda+a_{3}=0
\end{gathered}
$$

where

$$
\begin{gathered}
a_{1}=\frac{\Lambda}{S}+\frac{\phi S}{V} \\
a_{2}=\alpha^{2} \sigma^{2} V I+\frac{\phi \Lambda}{V}+\alpha^{2} S I \\
a_{3}=\frac{\alpha^{2} \sigma^{2} \Lambda V I}{S}+\frac{\phi \alpha^{2} S^{2} I}{V}+\phi \alpha^{2} \sigma S I
\end{gathered}
$$

By the Routh-Hurwitz criterion, the endemic equilibrium is stable if and only if

$$
a_{1}>0, a_{3}>0 \text { and } a_{1} a_{2}-a_{3}>0
$$

Clearly

and

$$
\begin{gathered}
a_{1}=\frac{\Lambda}{S}+\frac{\phi S}{V}>0 \\
a_{3}=\frac{\alpha^{2} \sigma^{2} \Lambda V I}{S}+\frac{\phi \alpha^{2} S^{2} I}{V}+\phi \alpha^{2} \sigma S I>0
\end{gathered}
$$

So the endemic equilibrium is stable if and only if $a_{1} a_{2}-a_{3}>0$.

i.e.,

$$
\left(\frac{\Lambda}{S}+\frac{\phi S}{V}\right)\left(\alpha^{2} \sigma^{2} V I+\frac{\phi \Lambda}{V}+\alpha^{2} S I\right)-\left(\frac{\alpha^{2} \sigma^{2} \Lambda V I}{S}+\frac{\phi \alpha^{2} S^{2} I}{V}+\phi \alpha^{2} \sigma S I\right)>0
$$

i.e.,

$\frac{\left(\left(\phi \alpha^{2} \sigma^{2}-\phi \alpha^{2} \sigma\right) I S^{2}+\alpha^{2} \Lambda S I\right) V^{2}+\phi \Lambda^{2} V+\phi^{2} \Lambda S^{2}}{S V^{2}}>0$

i.e.,

$$
\left(\left(\phi \alpha^{2} \sigma^{2}-\phi \alpha^{2} \sigma\right) I S^{2}+\alpha^{2} \Lambda S I\right) V^{2}+\phi \Lambda^{2} V+\phi^{2} \Lambda S^{2}>0
$$

where

$$
\begin{gathered}
\Lambda-\alpha S I-\phi S-\mu S=0 \\
\phi S-\mu V-\sigma \alpha V I=0 \\
\sigma \alpha V+\alpha S-\mu=0
\end{gathered}
$$




\section{Conclusion}

In this paper a new deterministic epidemic model is constructed and used to analyze the effect of a preventive vaccine on the transmission dynamics of an infectious disease. The model is thoroughly analyzed to investigate the stability. From the theoretical discussion and numerical simulations for the DFE, we see that if the parameters satisfy any of the equivalent conditions $\sigma<\sigma_{0}, \alpha<\alpha_{0}, \Lambda<\Lambda_{0}$ and $\phi>\phi_{0}$ then there is no epidemic. So, in the initial stage (when the number of infected individuals is not large), we shall control the epidemic successfully by controlling the parameters. Also for the endemic equilibrium we give a condition for stability (if there exist an endemic equilibrium).

\section{References}

[1]. Kermack, W.O., \& McKendrick, A.G. (1927). A contribution to the mathematical theory of epidemics. Proceedings of the Royal Society of London. Series A, 115, 700-721.

[2]. Driessche P.V.D. and Watmough J. (2002). Reproduction numbers and sub-threshold endemic equilibria for compartmental models of disease transmission. Mtahematical Bioscience, 180, 29-48.

[3]. Hethcote, H.W. (2000). The Mathematics of Infectious Diseases. Society for Industrial and Applied Mathematics, 42(4), 599-653.

[4]. Arino, J., McCluskey, C.C., \& Driessche, P.V.D. (2003). Global results for an epidemic model with vaccination that exhibits backward bifurcation. SIAM J. Appl. Math., 64, 260-276.

[5]. Buonomo, d'Onofrio, B. A., \& Lacitignola, D. (2008). Global stability of an SIR epidemic model with information dependent vaccination, Mathematical Biosciences, 216, 9-16.

[6]. Islam, M.S., Asaduzzaman, M., \& Mondal, M. N. I. (2012). Stability analysis at DFE of an epidemic model in the presence of a preventive vaccine. IOSR Journal of Mathematics, 3(2), 25-31.

[7]. Diekmann, O., Heesterbeek, J.A.P., \& Metz, J.S.J. (1990). On the definition and the Computation of the basic reproduction number ratio $\mathrm{R}_{0}$ in models for infectious diseases in heterogeneous population. J. Math. Bio 28, 365- 382.

[8]. Routh-Hurwitz Criterion. Retrieved from http://web.abo.fi/fak/mnf/mate/kurser/dynsyst/2009/R-Hcriteria.pdf 